\title{
Cultural Role: the Evolution of Traditional Martial Arts
}

\author{
Yue Lu \\ Chengdu Sports University, China \\ 1450637059@qq.com
}

\section{Key words: Traditional martial arts; Essential Characteristics; Rheological Properties}

\begin{abstract}
Using the research methods of literature and logical analysis, the article explains and analyzes the essential characteristic of Wushu. Through the four dimensions of space extrusion technique and action variation, role transformation, collision and integration of the moderate adjustment reasonable position of traditional Wushu in the perspective of disenchantment in the development process and abstract exploration and thinking of anxiety, and challenges the development and dissemination process, in the traditional martial arts "change" and "invariance" in the process of further discussion and consideration of the nature of the problem, find the deep cognition in the martial arts culture, and thinking of the traditional martial arts culture in the contemporary transformation in the development of the theory of cognition and path search.
\end{abstract}

\section{Introduction}

The significance of Wushu to the nation involves the dissemination and continuation of outstanding values, the promotion of traditional civilization genes, the optimization of the ideology of the country, the reform of the people's thinking mode, and the evolution of the customs and rituals of the nation. In the field of human civilization, and culture system of martial arts cultural context is a complex domain, not only refined in the controversial concept and discussion, because more characteristics in common with the unity of opposites as a cultural body show the. Re positioning it as a national culture may lead to its features, both of which are rheological arguments, and may also confirm their unchanging assertions. Therefore, with the help of aesthetic context, we can provide ideas for clarifying the development of traditional Wushu culture.

\section{The art of attack and defence in Wushu: the dominant cognition of traditional martial arts}

Squeeze the living space. To make the study more convincing argument, a questionnaire survey was conducted on the part of Chinese martial arts and martial arts experts practicing people. During the investigation, 200 questionnaires issued, which interviewed 180 popular martial arts practitioners and martial arts expert 20. People who practice martial arts for the masses to recover 180 questionnaires, the recovery rate of $100 \%$; 20 copies of the martial arts expert questionnaire, the recovery rate was $100 \%$. According to the preference of Wushu training groups, age and other factors, the removal of 15 invalid questionnaires, 185 valid questionnaires were collected, the effective recovery rate was $90.5 \% ; 20$ copies of expert questionnaires, the effective recovery rate of $100 \%$.

The traditional martial arts is "physical culture form a kind of implicit traditional culture," [1] is a unique symbol to combat characteristics, is the civilization factor rich traditional culture, is different from other sports and become a typical paradigm represents national culture. The traditional martial arts is the inheritance and development of national culture civilization gene, it not only contains abundant cultural connotation and technology system, and also become the foreign exchange "brand", and become a cultural symbol of the world wide recognition. Interpretation of martial arts is not entirely a sport, it may also enjoy its own independence of the sky, so that it is more conducive to its development". in this sense, how to recognize the traditional martial arts, or the martial arts belong to which areas of the category, perhaps many martial arts researchers want to know. 
Table 1: Traditional martial arts living space cognitive statistics

\begin{tabular}{c|c|c}
\hline Influence Gene & Quantity & Proportion (\%) \\
\hline Outland Culture & 30 & 15.71 \\
\hline Native Culture & 80 & 40.88 \\
\hline Total & 110 & 100.00 \\
\hline
\end{tabular}

The alienation of technical action. Under the infiltration of western sports thoughts, the traditional sports culture ecology of our country has developed difficultly, and the traditional culture represented by traditional Wushu is facing the risk of being weakened. However, it is inevitable that the cultural composition of competitive sports is highly westernized, which makes the cultural soil of traditional Wushu more barren.

In addition, in the western and local conflicts with media under the premise of Western and local attention has been unbalanced, resulting in human cognition of the culture of the traditional martial arts can only stay in the surface meaning, this is undoubtedly in the level of development of culture is relatively dwarfed by local culture, makes the traditional martial Arts in the future development of relative weaken.

\section{The culture: the implicit interpretation of traditional Wushu}

The moderate adjustment of role transformation. Through the questionnaires of 100 popular martial arts practitioners and 20 experts in the analysis of the results, $20.89 \%$ of the respondents think that the traditional Wushu should follow the overall conservative mode, but also its skills, but also to protect the important paradigm of traditional culture; and 50.75\% practitioners think that the development of traditional Wushu should follow the development of western style the traditional martial arts, tend to art.

"Martial arts symbols, like a sponge, contain a wide variety of ingredients, absorb a wide variety of knowledge, and allow and default various interpretations." ${ }^{[3]}$ the traditional martial arts in the development process with the historical, perhaps to absorb and receive foreign culture, may have thought the bottleneck of traditional thinking to break their own bonds, the establishment of the system of new development theory, grasp the reasonable position of the Conservatives and the Westernization, out of its dilemma.

Table 2: Statistics of traditional martial arts role cognition

\begin{tabular}{c|c|c}
\hline & Quantity & Proportion (\%) \\
\hline Complete Westernization & 80 & 80 \\
\hline Conservative & 20 & 20 \\
\hline Total & 100 & 100.00 \\
\hline
\end{tabular}

Reasonable positioning of blending collisions. In the martial arts culture changed and unchanged, creating a cultural exchange platform to speak, while retaining the inherent quality of local culture, and other elements of fusion culture continue to absorb, should reflect the continuity of tradition and map age and nationality.

When the traditional martial arts of Westernization will be questioned for cultural betrayal, but when people stop conformism and other excellent cultures, collision, and traditional martial arts in the development of the embarrassing situation and always on tenterhooks. In this kind of contradiction in the development, when we take the perspective of culture cognition of traditional martial arts, the accurate positioning of the traditional martial arts, and explore its essential characteristics in the flow process, may be found behind more profound meaning. 


\section{Conclusion}

Mills, a famous American critical sociologist, said: "the good definition should be the result of changing the argument of terminology to the different views of facts, thus bringing about the arguments needed for further research."[4] Then, it may be possible to provide more paradigms and academic help for the study of martial arts culture. To take reasonable measures, is not the "Westernization", not "overall conservative", this is a series of problems and demonstration to explore it in different ideological dimension, martial arts experts and scholars of theoretical clues and discussion related research to clarify the context of the development of martial arts culture.

\section{Reference}

[1]Chen Zhenyong, Guo Zhiyu. The concept of international communication development of Wushu movement. Interaction of body culture [J]. Journal of Shanghai Institute of Physical Education, 2005 (6): 51-54.

[2].Niu Xiaoben cattle martial arts clothing culture of [J]. sports culture daokan, 2010,33 (10): 103-105.

[3]Dai Guobin. Martial arts: body culture, [M]., people's Sports Publishing House, 2011,6:6.

[4]Chen Zhenyong. Research on regional Wushu Culture in anthropological view [J]. Journal of Chengdu Sport University, 2016,42 (2): 54-55. 